\title{
Lapurdum
}

LAPURDUM Euskal ikerketen aldizkaria | Revue d'études basques |

Revista de estudios vascos | Basque studies review

$7 \mid 2002$

Numéro VII

\section{Les héritières de la maison au Pays Basque au XIXe siècle}

\author{
Marie-Pierre Arrizabalaga
}

\section{OpenEdition \\ Journals}

Édition électronique

URL : http://journals.openedition.org/lapurdum/955

DOI : 10.4000/lapurdum.955

ISSN : 1965-0655

\section{Éditeur}

IKER

Édition imprimée

Date de publication : 1 octobre 2002

Pagination : $37-55$

ISBN : 2-86781-321-2

ISSN : $1273-3830$

Référence électronique

Marie-Pierre Arrizabalaga, "Les héritières de la maison au Pays Basque au XIXe siècle », Lapurdum [En ligne], 7 | 2002, mis en ligne le 01 juin 2009, consulté le 19 avril 2019. URL : http:// journals.openedition.org/lapurdum/955; DOI : 10.4000/lapurdum.955 


\title{
Les héritières de la maison au Pays Basque au XIXe siècle
}

\author{
Marie-Pierre ARRIZABALAGA ${ }^{1}$
}

Université de Cergy-Pontoise

IKER UMR 5478 CNRS

Les coutumes successorales au Pays Basque dans l'Ancien Régime étaient unique en Europe non parce qu'elles permettaient aux chefs de famille de léguer tous les biens de famille aux aînés (car c'était le cas de nombreuses régions coutumières en France ${ }^{2}$ ), mais parce que ce système de l'héritage unique, celui de la primogéniture qui favorisait l'aîné des enfants, ne faisait aucune distinction entre les garçons premiers nés et les filles premières nées. Selon le droit coutumier basque, l'aîné, qu'il soit un garçon ou une fille, devenait l'héritier légal de la maison et de toutes les terres, forcé(e) ensuite de dédommager plus ou moins équitablement les cohéritiers, filles et garçons, qui dès lors quittaient la maison et allaient se placer ailleurs. Ainsi, les filles aînées avaient autant de chances que les fils aînés d'hériter du patrimoine familial. Ce système n'a pas d'équivalence dans les Pyrénées (ni même en France ou en Europe). Les Béarnais préféraient transmettre la succession de la maison aux fils aînés en priorité ou aux fils cadets, lorsque l'aîné des enfants était une fille ${ }^{3}$. De même, dans les Baronnies, c'était l'aîné des garçons qui généralement héritait du patrimoine familial ${ }^{4}$. Enfin, en Catalogne, l'héritage était transmis à un fils, qu'il

\footnotetext{
${ }^{1}$ Marie-Pierre Arrizabalaga est Maître de Conférences à l'Université de Cergy-Pontoise où elle enseigne depuis cinq ans. Elle a obtenu deux doctorats, le premier en 1994 au département d'Histoire de l'Université de Californie à Davis aux Etats-Unis et le second en 1998 à l'Ecole des Hautes Etudes en Sciences Sociales à Paris. Elle consacre sa recherche et ses publications françaises et étrangères à l'étude de la famille, des structures familiales, des successions et ses modalités, du sort des héritiers et des cadets et de l'émigration des Basques au XIXe siècle.

2 Voir les typologies des modes de transmission présentées par Jean Yver, Essai de géographie coutumière (Paris: Editions Sirey, 1966); Emmanuel Le Roy Ladurie, "Système de la coutume. Structures familiales et coutume d'héritage en France au XVIe siècle", Annales E.S.C., 4-5, 1972, 825-846; André Burguière, "Pour une typologie des formes d'organisation domestique de l'Europe moderne (XVle - XIXe siècles), Annales E.S.C., 3, 1986, 639-655; Louis AssierAndrieu, "Coutume savante et droit rustique. Sur la légalité paysanne", Etudes Rurales, $103-$ 104, 1986, 105-137; Pierre Lamaison, "La diversité des modes de transmission: une géographie tenace", Etudes Rurales, 110-112, 1988, 119-175; Bernard Derouet, "Pratiques successorales et rapport à la terre: les sociétés paysannes d'Ancien Régime", Annales E.S.C., 1, 1989, 173-206.

3 Prochainement sera publié l'ouvrage de Christine Lacanette-Pommel, Les Béarnais et le Code Civil. Etude des pratiques successorales et matrimoniales dans les Pyrénées (1789-1840), Toulouse, thèse de troisième cycle en droit, Université des Sciences Sociales, 1998.

"Antoinette Fauve-Chamoux, "Les frontières de l'auto-régulation paysanne: croissance et famillesouche", Revue de la Bibliothèque Nationale, 1993, 50, p. 38-47 \& "Les structures familiales au royaume des familles-souches: Esparros", Annales ESC, 1984, XXXIX, 3, p. 513-528. Voir aussi l'ouvrage sur les Baronnies, ouvrage collectif dirigé par I. Chiva \& J. Goy, Les Baronnies des Pyrénées. Maisons, mode de vie, société, Paris, EHESS, 1981, ainsi que Les Baronnies des Pyrénées. Maisons, espace, famille, Paris, EHESS, 1986.
} 
fût l'aîné ou le cadet des enfants, mais quasiment jamais à une fille'. Ainsi, le cas basque de l'aînesse intégrale ou de primogéniture sans distinction entre les sexes était-il unique dans le Sud de la France ${ }^{2}$. Dans le Nord de la France, les familles respectaient le partage égalitaire entre les héritiers, généralement entre les garçons $3^{3}$.

Le système de l'héritage unique en faveur de l'aîné, fille ou garçon, ou l'aînesse intégrale, si traditionnel au Pays Basque, a jusqu'au XIXe siècle considérablement influencé les structures de la famille et a longtemps été respecté par les familles afin de préserver intact le patrimoine familial et surtout la maison ("etche" en Basque). Ces pratiques étaient courantes dès le Moyen Age et peut-être même avant, mais elles furent codifiées pendant la période moderne dans le but d'institutionnaliser le système et de légaliser les pratiques familiales traditionnelles. Ainsi, les coutumes basques ont permis aux familles de légaliser l'héritage unique favorisant le fils aîné ou la fille aînée, selon le sexe de l'enfant premier né, un système qui survécut au cours du XIXe siècle en dépit de la Révolution française et du Code Civil de 1804 qui imposait l'héritage égalitaire entre les enfants, filles ou garçons, premiers ou derniers nés. Le patrimoine familial était par conséquent transmis au premier né des enfants, le fils aîné (succession patrilinéaire) ou la fille aînée (succession matrilinéaire), qui se devait de dédommager plus ou moins équitablement les cohéritiers en les dotant d'une somme d'argent qui leur permettait de se marier et de "se caser". Le système ainsi élaboré démontrait que pour les familles, le patrimoine et la maison devaient impérativement rester intacts, transmis à un seul descendant qui dès lors formait un groupe familial élargi avec ses parents, son conjoint, et ses enfants (et parfois même des cohéritiers célibataires), vivant sous le même toit, travaillant ensemble et partageant le fruit de leur labeur collectif. Chaque famille était ainsi composée de plusieurs générations cohabitant sous le même toit et partageant toutes les activités et les obligations liées à la maison et à l'exploitation. Les structures de ces familles étaient donc complexes. C'étaient celles de la famille traditionnelle basque : la famille souche.

Avec l'obligation de partage égalitaire entre les cohéritiers imposé avec la mise en place du Code Civil en 1804, il était logique que les systèmes inégalitaires de l'héritage unique en France dont celui de l'aînesse intégrale favorisant l'aîné des enfants, fille ou garçon, disparaissent sur tout le territoire français au cours du XIXe siècle. Or, de nombreuses études sur les anciennes provinces pyrénéennes ont démontré le contraire. Le Code Civil a mis beaucoup de temps à s'imposer dans les campagnes françaises et l'héritage unique, bien que transformé, a survécu dans les provinces du Sud de la France et dans les

\footnotetext{
Voir les ouvrages de Louis Assier-Landrieu, Le peuple et la loi. Anthropologie historique des droits paysans en Catalogne française, Paris, Librairie de droit et de jurisprudence, 1987 \& Coutume et rapports sociaux. Etude anthropologique des communautés paysannes du Capcir, Paris, Editions du CNRS, 1981.

2 Concernant les études des systèmes successoraux dans le Sud Ouest de la France, consulter les ouvrages suivants: Jacques Poumarède, Les Successions dans le Sud-Ouest de la France au Moyen Age, Paris, Presses universitaires de France, 1972; Anne Zink, L'Héritier de la maison. Géographie coutumières du Sud-Ouest de la France sous l'Ancien Régime, Paris, EHESS, 1985; Maïté Lafourcade, Mariages en Labourd sous l'Ancien Régime. Les contrats de mariage du Pays de Labourd sous le règne de Louis XVI (étude juridique et sociologique), Bilbao, Universidad del Pais Vasco, 1989.

3 Pour une typologie des modalités successorales en France dans l'Ancien Régime avec une bibliographie complète, voir l'article de Bemard Derouet, "Pratiques successorales et rapport à la terre: les sociétés paysannes d'Ancien Régime", Annales E.S.C., 1, 1989, 173-206.
} 
régions montagneuses frontalières en dépit des nouvelles lois. En fait, les familles ont élaboré de nouvelles stratégies afin de contourner ces lois et permettre à un seul enfant d'hériter de la maison et du patrimoine pour préserver ainsi intacts le patrimoine et la maison. Les familles ont dû faire preuve d'adaptabilité face au nouveau régime successoral égalitaire. Elles continuaient à transmettre le patrimoine familial et la maison à un seul enfant tout le long du siècle, s'efforçant de dédommager les cohéritiers en les dotant suffisamment afin qu'ils n'exigent pas leur part légale de l'héritage et quittent la maison familiale, satisfaits de leur dot. Ces observations démontraient clairement que les pratiques successorales anciennes remodelées au XIXe siècle au Pays Basque n'existaient pas seulement en raison de leur codification à l'époque moderne, mais qu'elles étaient réellement inhérentes à la culture des Basques qui n'envisageaient la pérennité de la famille que par la survie de la maison et de son patrimoine. Cette survie était selon eux dépendante de l'indivision d'une génération à l'autre. Ces valeurs familiales étaient si inhérentes à la culture basque que les coutumes écrites (les Fors) ne donnaient en fait qu'une existence légale à ces pratiques, le but étant depuis des siècles de préserver la maison et les terres intactes afin d'assurer la viabilité de la maison et l'équilibre écodémographique des villages ${ }^{1}$.

Contrairement à ce qu'a affirmé Frédéric Le Play ${ }^{2}$ concernant les effets négatifs du Code Civil sur les successions et la famille souche, le Code Civil n'a pas eu pour effet la fragmentation des propriétés au Pays Basque au XIXe siècle, la mise en vente progressive des petites propriétés, et la disparition de la famille souche ${ }^{3}$. Effectivement, les familles ont continué à transmettre le patrimoine à un seul enfant en dépit des nouvelles lois égalitaires pour réserver la plus grande part des biens à cet héritier unique. Elles s'efforçaient ainsi d'éviter le partage. Cependant, en dépit de tous les efforts, le Code Civil et les nouvelles conditions économiques liées à l'industrialisation les ont contraintes à

\footnotetext{
Les communes basques étaient généralement petites et leur potentiel économique limité en raison de la présence de montagnes, souvent peu exploitables. Chaque exploitation familiale était pour la plupart petite ou moyenne et ne pouvait survivre que si elle restait intacte et indivis. En raison de ces conditions économiques limitées, les familles avaient établi des pratiques successorales qui empêchaient le partage du patrimoine et permettaient à l'aîné des enfants, fille ou garçon, comme nous l'avons vu ci-dessus, d'hériter de la maison et du patrimoine el ainsi assurer la survie de la lignée et de l'exploitation d'une génération à l'autre. Les familles avaient pour responsabilité première de préserver ce patrimoine intact et de le transmettre intact ou amélioré à la génération suivante. Non seulement la survie de la famille en dépendait, mais aussi l'équilibre économique des communes. Ces demières ne pouvaient se permettre de voir les fermes se multiplier et pour cela, les familles devaient trouver toutes les solutions possibles, non seulement pour transmettre le patrimoine à un seul héritier, mais aussi pour assurer l'avenir des cohéritiers de l'héritier unique grâce à un dédommagement financier qui facilitait leur installation dans la commune ou ailleurs.

${ }^{2}$ Frédéric Le Play souligne les effets négatifs du Code Civil qui oblige les parents à partager leur biens 'équitablement entre tous les enfants. II démontre qu'à cause de cela, les familles devaient souvent vendre le patrimoine pour pouvoir dédommager tous les héritiers équitablement, multipliant ainsi le nombre de petits propriétaires qui ne pouvaient vivre de leur terre et qui donc vendaient leurs biens et quittaient le village. De cette situation, il en résulte la disparition progressive de nombreuses maisons et la remise en cause de la famille souche, bastion de la stabilité économique et démographique des villages pyrénéens. Frédéric Le Play, L'organisation de de la famille selon le vrai modele signalé par l'histoire de toutes les races et de tous les temps, Paris, Tequi, 1871. \& La réforme sociale en France déduite de l'organisation comparée des peuples européens, Paris, Dentu, 1878.

3 Voir le débat concernant les effets du Code Civil dans l'article de Marie-Pierre Amizabalaga, "The stem family in the French Basque Country: Sare in the nineteenth century", Journal of Family History, vol. 22, 1, 1997, 50-69 \& "Structures familiales et destins migratoires à Sare au XIXe siècle", Lapurdum, II, 1997, 237-255.
} 
modifier leurs pratiques successorales traditionnelles. Elles devaient à présent tenir compte de plusieurs paramètres : le Code Civil tout particulièrement car il imposait l'égalité des droits entre les cohéritiers, mais aussi l'industrialisation, l'urbanisation et l'émigration qui éloignaient les jeunes basques de leur village et leur offraient de nouvelles opportunités. L'ouverture du marché de l'emploi en France et à l'étranger a transformé remarquablement les modalités de transmission du patrimoine notamment à partir de la seconde moitié du XIXe siècle. De nombreux héritiers potentiels s'éloignaient de leur village, temporairement dans un premier temps, puis définitivement, obligeant les familles à choisir l'héritier unique parmi les cadets, filles ou garçons, une situation qui a ensuite contraint de plus en plus de familles à choisir leur héritier parmi les filles cadettes, moins nombreuses à quitter le village et plus disponibles pour assurer la tradition familiale. Ces filles n'étaient pas forcément les aînées, mais aussi et surtout des cadettes, qui ne faillissaient pas à leur devoir et remplaçaient les frères absents. Ce qui prévalait, c'était qu'un des enfants succède aux parents, devienne l'héritier unique et assure l'indivisibilité du patrimoine d'une génération à l'autre. Les filles, comme les garçons, semblaient assumer ces responsabilités de manière tout à fait satisfaisante pour les familles. Tant qu'un enfant acceptait d'assurer cette tâche, le sexe et le rang de naissance de cet héritier importaient peu.

Le but de la présente démonstration est précisément d'analyser brièvement les coutumes anciennes pratiquées au Pays Basque jusqu'à récemment, tout particulièrement les modalités de l'aînesse intégrale afin de démontrer que, selon le droit coutumier, les filles aînées avaient autant de chance que les fils aînés d'hériter de la maison et de son patrimoine. Cependant, au cours du XIXe siècle, avec la mise en place du Code Civil et les phénomènes d'industrialisation, d'urbanisation et d'émigration, les familles basques ont dû s'adapter et élaborer de nouvelles stratégies afin de préserver la maison et les terres sans jamais les partager entre les cohéritiers. Dans la pratique, les familles n'hésitaient pas à choisir l'héritier parmi les filles, aînées ou cadettes, qui dès lors récupéraient la maison et les terres et assuraient la succession. Dans quelles circonstances les familles ont-elles transmis leur patrimoine à un seul héritier, souvent une fille aînée ou cadette, et quelles en étaient les conséquences sur les pratiques familiales?

\section{Les coutumes basques sous l'Ancien Régime}

Les coutumes basques de l'Ancien Régime se composaient de lois familiales et communautaires qui correspondaient à des règles sociales, économiques et politiques établies par les chefs de famille, propriétaires des maisons dans le village, dans le but de garantir la stabilité collective et l'harmonie sociale au sein des familles et des communes. L'héritage unique était un des bastions de ces règles car il permettait aux familles de préserver les maisons intactes, assurait la survie économique des exploitations, pour la plupart de taille moyenne ou petite, et garantissait ainsi l'équilibre démographique et économique des familles et des communes. Jusqu'au XIXe siècle, chaque couple avait en moyenne trois enfants adultes survivants. Tandis que l'aîné, fille ou garçon, héritait du patrimoine familial, le second, doté, épousait un héritier ou une héritière, et le troisième restait célibataire dans la maison familiale. En 1454, le roi Charles VII ordonna que toutes les coutumes locales sur le territoire français soient écrites afin que toutes les pratiques familiales et locales, privées ou 
publiques, deviennent officiellement institutionnalisées et légales. Suite à cet ordre, des officiers du roi furent chargés de produire des documents écrits et officiels sur les coutumes des trois provinces basques, ce qui fut fait pour le Labourd $^{1}$, la Basse-Navarre ${ }^{2}$, et la Soule ${ }^{3}$, tous publiés pour la première fois à la fin du XVe siècle ou le début du XVIe siècle. Jusqu'à la Révolution française, ces coutumes ne furent jamais remises en cause par les autorités locales, régionales ou nationales car elles préservaient la paix dans les familles, la stabilité économique des communes, et l'harmonie dans toutes les provinces du royaume.

Les articles coutumiers qui nous intéressent ici sont ceux relatifs aux pratiques familiales, qui déterminaient les structures de la famille et son fonctionnement. Les quatre règles essentielles à la survie de la famille concernaient le choix de l'héritier (celui de l'aînesse intégrale), le choix du conjoint de l'héritier, le moment et les conditions choisis pour le mariage de l'héritier, et les droits des cohéritiers. Selon les coutumes, les familles n'avaient pas réellement la possibilité de choisir l'héritier parmi tous leurs enfants car l'aîné des enfants, fille ou garçon, détenait des droits d'aînesse intégrale qui ne pouvaient lui être retirés, sauf en cas de désaccord, de désobéissance parentale ou d'impossibilité physique ou mentale. Effectivement, les coutumes protégeaient les droits des aînés sur la maison et le patrimoine familial et garantissaient leur supériorité sur ses frères et sœurs cadets. Lorsque l'héritier se remariait, les droits d'aînesse étaient réservés à l'aîné des enfants du premier mariage, même si les enfants du premier mariage étaient tous des filles et que ceux du second mariage comprenaient des garçons. Ainsi, selon les coutumes ancestrales basques, les filles aînées étaient considérées de la même manière que les fils aînés, jouissaient des mêmes droits que les fils aînés, et jouaient un rôle aussi important dans la maison. Elles participaient aux décisions concernant l'exploitation familiale et son devenir, et avaient une responsabilité toute aussi importante sur le choix de l'héritier et la transmission du patrimoine d'une génération à l'autre.

Bien que propriétaire de ses biens, l'héritier ne pouvait disposer du patrimoine familial comme bon lui semblait. Il ne pouvait choisir l'héritier qu'il souhaitait, son rôle se limitant à celui de responsable ou de régisseur des biens, se devant de préserver la paix dans le foyer en transmettant la maison et lcs terres intactes à l'aîné des enfants. Ainsi, ils étaient propriétaires des biens immobiliers que leurs parents leur avaient transmis, mais ne pouvaient en disposer librement. Pour vendre, ils devaient obtenir le consentement des parents (s'ils vivaient encore) et celui des cohéritiers ascendants, collatéraux et descendants (le cas échéant). Tant qu'ils ne percevaient pas de dot ou de dédommagement pour leur part de l'héritage, les cohéritiers préservaient leurs droits de décision sur le fonctionnement de l'exploitation familiale et sur le devenir du patrimoine familial. L'héritier et sa famille se devaient de vivre et de travailler avec les parents donateurs. Ils partageaient les responsabilités, les dépenses et les revenus de l'exploitation. L'association, ainsi créée, était une "association de coseigneurie", où les héritiers parents et enfants participaient

' Voir textes publiés dans Coûtumes générales, gardées et observées au Païs et baillage de Labourt, et reffort d'icelui, Bordeaux, J-B Lacomée, 1760.

2 Voir textes publiés et commentés par Jean Goyeneche, For et coutumes de Basse-Navarre, Bayonne, Elkar, 1985.

3 Voir textes publiés par Michel Grosclaude, La Coutume de la Soule. Traduction, notes et commentaires, Saint-Etienne-de-Baïgorry, Izpegi, 1993. 
ensemble à l'administration du patrimoine familial, partageaient les revenus de l'exploitation et cohabitaient dans la maison que l'héritier avait héritée. Les structures de la famille étaient donc complexes, deux générations d'individus vivant ensemble avec parfois des cohéritiers célibataires à chaque génération. Cette structure était celle de la famille souche qui s'imposa pendant des siècles et qui, comme nous le verrons, perdura pendant tout le XIXe siècle, voire même pendant le XXe siècle.

Les responsabilités qui pesaient sur l'héritier pour préserver la maison et le patrimoine intacts et les transmettre indivis à la génération suivante étaient telles que les parents donateurs se devaient de choisir le bon moment et la bonne personne pour leur succéder. Ils n'avaient pas le choix quant à l'héritier, le premier né, fille ou garçon, détenant tous les droits sur la succession. Néanmoins, ils devaient choisir le moment du mariage de l'héritier ou de l'héritière, son conjoint, et la manière la plus sûre pour assurer la survie de la maison et sa transmission à la génération suivante. L'héritier devait se marier suffisamment jeune pour avoir une descendance mais pas trop jeune pour ne pas avoir un trop grand nombre d'enfants à dédommager ensuite. L'héritier se mariait généralement assez tard, plus tard parfois que certains de ses cohéritiers et après le départ de certains d'entre eux de la maison. Ces deux dernières conditions étaient nécessaires pour qu'il y ait suffisamment de place pour abriter l'héritier et sa famille et que les revenus puissent permettre à tous les résidents de la maison de survivre. Pour le départ des cohéritiers, la famille prévoyait une aide ou une dot, grâce à la dot du conjoint du jeune héritier. L'argent se faisant rare à l'époque, certains cohéritiers étaient autorisés à vivre célibataire dans la maison familiale. Ces derniers, non dotés, préservaient un "droit de chaise" et participaient quelque peu aux décisions familiales. C'est pourquoi le choix du conjoint de l'héritier était aussi important. Il devait vivre avec ses beaux-parents, accepter de leur obéir, et se soumettre à l'autorité de son conjoint. En outre, ce conjoint devait apporter une dot suffisamment importante pour dédommager au mieux un ou plusieurs cohéritiers qui dès lors quittaient la maison et permettaient à l'héritier ou à l'héritière de contrôler l'intégralité du patrimoine hérité. Ces règles semblaient être rigoureusement appliquées jusqu'à la Révolution française ${ }^{1}$, mais furent remises en cause par le Code Civil qui imposait une égalité stricte entre les cohéritiers. Quelles furent donc les conséquences du Code Civil sur ces pratiques au XIXe siècle, notamment sur l'aînesse intégrale et les modalités de transmission du patrimoine?

\section{Le choix de l'héritier}

Dans notre analyse des pratiques familiales au Pays Basque au XIXe siècle; nous avons élaboré une méthode basée sur les reconstitutions de famille, le but étant de déterminer le nombre de descendants, le choix de l'héritier, et les modalités de transmission du patrimoine. Pour cela, nous avons choisi 120 couples qui se sont mariés dans six villages différents du Pays Basque : un en Labourd (Sare), quatre en Basse Navarre (Les Aldudes, Mendive, Isturits et

\footnotetext{
1 Voir les études faites sur les pratiques successorales au Pays Basque sous l'Ancien Régime à partir de l'analyse des contrats de mariage et des testaments par Anne Zink, L'Héritier de la maison. Géographie coutumières du Sud-Ouest de la France sous l'Ancien Régime, Paris, EHESS, 1985 \& Maïte Lafourcade, Mariages en Labourd sous l'Ancien Régime. Les contrats de mariage du Pays de Labourd sous le règne de Louis XVI (étude juridique et sociologique), Bilbao, Universidad del Pais Vasco, 1989.
} 
Amendeuix) et un en Soule (Alçay) ${ }^{1}$. Nous avons donc sélectionné vingt couples par village, tous mariés dans la première décennie du XIXe siècle. Leurs enfants sont nés pendant la période se situant entre 1830 et 1860 et leurs grands enfants entre 1860 et 1900 . Pour reconstituer ces familles, nous avons utilisé l'état civil de quasiment tous les villages du Pays Basque, les chefs-lieux de canton, Bayonne et Pau. Au total, nous avons reconstitué les destins d'environ 3000 personnes. Ensuite, nous avons consulté le Cadastre, l'enregistrement et tout particulièrement les mutations après décès, les archives notariales nous étant apparues incomplètes, notamment pour certaines communes telles que Sare ${ }^{2}$. Grâce à ces sources, nous nous sommes efforcés de déterminer le choix de l'héritier à chaque génération, le destin des cohéritiers, et les modalités de transmission du patrimoine des familles propriétaires pendant trois générations (entre 1800 et le début du XXe siècle).

L'analyse des 120 familles ainsi reconstituées fait apparaître qu'effectivement l'héritage unique, bien qu'illégal après l'instauration du Code Civil en 1804, était toujours une pratique courante au Pays Basque pendant toute la période étudiée. Néanmoins, par rapport à l'Ancien Régime, les modalités de cet héritage unique ont considérablement évolué permettant à davantage de filles, aînées ou cadettes, d'hériter du patrimoine familial. L'analyse ci-dessous montrera que les familles dans certains villages respectaient rigoureusement les coutumes ancestrales de l'aînesse intégrale, transmettant leur patrimoine au premier né des enfants, garçon ou fille, tandis que dans d'autres villages, les familles transmettaient de plus en plus souvent leur patrimoine à un enfant cadet, notamment des filles cadettes, comme les tableaux 1 et 2 ci-dessous le montrent.

À travers l'analyse des 120 reconstitutions de famille, nous avons pu identifier les comportements des 120 couples (première génération), ceux de leurs enfants (héritiers ou non héritiers) dans la première moitié du siècle, jusque 1860 (seconde génération) et ceux de leurs descendants après 1860 et jusqu'au début du XXe siècle (troisième génération). Nous avons constaté que pendant la totalité du XIXe siècle, les familles ont continué à transmettre la maison familiale et les terres à un seul enfant, en dépit de la mise en place du Code Civil et de l'obligation d'égalité de transmission auxquelles les parents devaient se soumettre à l'égard de tous leurs enfants. Cependant les modalités de cette transmission ont considérablement évolué au cours du siècle, comme nous allons le démontrer.

Dans la première moitié du XIXe siècle, la très grande majorité des 120 couples a transmis les biens à l'enfant premier né, fille ou garçon, comme dans les coutumes, une transmission qui avait généralement lieu entre 1830 et 1860 , en partie lors de la signature du contrat de mariage et le reste ensuite, voire au

\footnotetext{
Le choix des villages fut déterminé par rapport à la qualité des sources. Pour pouvoir reconstituer les familles, il fallait que l'état civil du village choisi soit complet, ainsi que celui des villages environnants. Un grand nombre de villages, notamment en Soule, faisait apparaître des lacunes importantes. Nous avons donc choisi davantage de villages de Basse Navarre. En outre, nous souhaitions comparer les comportements des populations des villages de montagne à ceux des villages de plaine, de préférence éloignés des villes côtières.

2 Après avoir consulté les index des archives notariales de la commune d'Espelette, nous nous sommes rendu compte que tous les contrats de mariage et les testaments concernant les familles de Sare et établis au XIXe siècle étaient manquants. Ils ont systématiquement été retirés des archives et demeurent introuvables à ce jour. Ils ont probablement été détruits.
} 
moment du décès des deux parents donateurs. Selon les données que nous avons récoltées, les deux tiers des héritiers étaient choisis parmi les enfants premiers nés : 21 (soit 52,5\%) étaient des filles aînées et 19 (soit 47,5\%) des fills aînés (voir Tableau 1). Ainsi, dans la première moitié du XIXe siècle, les familles basques s'efforçaient de transmettre la maison et les terres à un enfant et ce dernier était généralement le premier né des enfants, fille ou garçon, comme les pratiques ancestrales et les coutumes le préconisaient. L'aînesse intégrale était de ce fait toujours une réalité dans la première moitié du XIXe siècle, un système unique en France à la fois pendant l'Ancien Régime et après la Révolution française. En effet, les filles aînées étaient même un peu plus nombreuses que les fils aînés à hériter, ces derniers n'hésitant pas à quitter la maison familiale pour épouser une héritière ou à émigrer. Parmi les 20 héritiers cadets, la plupart était des hommes, soit 12 hommes (voir Tableau 1). Ces cadets ont hérité du patrimoine familial car l'ainé de la famille a choisi un autre destin : cinq fîls aînés ont épousé des héritières, deux sont restés célibataires dans la maison familiale (ne pouvant ou ne souhaitant pas assumer la responsabilité de la transmission), et cinq ont émigré en Amérique. Parmi les cinq filles aînées qui n'ont pas hérité, une a épousé un artisan aisé, une autre a hérité du patrimoine d'une autre lignée de la famille sans descendance, une est entrée au couvent, et enfin, quatre autres ont eu des enfants hors mariage et se sont probablement mariées sans le consentement de leurs parents. Qu'est-ce qui explique ces comportements différentiés ?

Tableau 1

L'aînesse intégrale dans la première moitié du XIXe siècle : seconde génération

\begin{tabular}{|c|c|c|c|}
\hline Rang de naissance & Fille & Fils & Total \\
\hline Aîné & $21 \quad(52,5 \%)$ & $19 \quad(47,5 \%)$ & $40 \quad(66,7 \%)$ \\
\hline Cadet & $8(40,0 \%)$ & $12(60,0 \%)$ & $20(33,3 \%)$ \\
\hline Total & $29(48,3 \%)$ & $31 \quad(51,7 \%)$ & $60(100 \%)$ \\
\hline
\end{tabular}

Dès la première moitié du XIXe siècle, le développement économique, l'industrialisation, l'urbanisation et l'émigration ont permis à quelques héritiers légitimes, les premiers nés des enfants, d'envisager un destin individuel autre que celui d'héritier. Ils ont pour certains épousé des héritiers ou des héritières, souvent plus aisés, ou ont émigré en Amérique. Quoi qu'il en soit, les héritiers désignés ne pouvaient en aucun cas remettre en cause l'autorité parentale, comme dans l'Ancien Régime, car ils n'étaient toujours pas libres d'épouser la personne de leur choix sans le consentement des parents. Ainsi, les pratiques ancestrales perduraient dans la première moitié du XIXe siècle avec notamment l'héritage unique (dans tous les cas), l'aînesse intégrale (dans les deux tiers des cas), et le consentement parental nécessaire pour se marier et hériter. Ces pratiques démontraient une volonté déterminée et une capacité infaillible à assurer la continuité des traditions et de la succession en dépit des nouvelles lois égalitaires. Néanmoins, de nouvelles pratiques et de nouveaux comportements ont fait leur apparition. Dans ce cas, ils obtenaient une compensation financière conséquente, une dot souvent élevée pour le dédommagement de tous les droits d'héritier. Ils se mariaient à un plus jeune âge que s'ils attendaient d'hériter de la maison et des terres après le départ de quelques cadets. Grâce à la dot élevée, ils épousaient des héritières souvent aisées, propriétaires de biens plus importants que ceux de leurs parents. Par ailleurs, les autres s'en allaient avec leur dot, 
l'industrialisation en France et en Amérique, couplée à une plus grande liberté de mouvement après la Révolution Française, les détournant des obligations d'héritier de la maison. La plupart allaient chercher fortune en Amérique, leur dédommagement leur permettant de s'y installer relativement confortablement. Dès la première moitié du XIXe siècle, nous observons que des héritiers étaient prêts à renoncer à leurs droits d'héritier afin de jouir d'une plus grande liberté professionnelle (pour les émigrants) ou matrimoniale (pour les femmes ayant des enfants illégitimes). Ces quelques cas, relativement rares dans la première moitié du XIXe siècle, sont révélateurs de changements dans les comportements des Basques dans la seconde moitié du XIXe siècle. De quelle manière ces nouveaux comportements ont-ils évolué dans la seconde moitié du XIXe siècle?

Les hommes et femmes qui ont hérité du patrimoine de leurs parents entre 1830 et 1860 ou qui ont épousé des héritières ou des héritiers dans la première moitié du siècle ont comme leurs ancêtres transmis la maison familiale et les terres à un seul enfant après 1860, reproduisant ainsi les pratiques de leurs parents en matière d'héritage unique. Cependant, ces familles se ne limitaient pas à l'aînesse intégrale ou à la transmission du patrimoine à l'aîné des enfants, fille ou garçon. Au lieu de cela, ils choisissaient leur héritier unique parmi un de leurs enfants, aîné ou cadet, fille ou garçon, de telle sorte que, dans la seconde moitié du XIXe siècle, les pratiques ancestrales de l'aînesse intégrale n'étaient plus aussi rigoureusement appliquées. Effectivement, à peine plus de la moitié des familles a transmis le patrimoine familial à l'enfant premier né, fille ou garçon, soit au total 47 familles sur 86 (54,7\% des cas). Parmi les 47 aînés, 30 étaient des filles (soit $63,8 \%$ ) et 17 seulement des garçons (soit $36,2 \%$ ) (voir Tableau 2). Les données ici présentées démontrent une évolution remarquable dans les modalités des pratiques successorales dans la première moitié du siècle car les filles aînées étaient deux fois plus nombreuses à hériter du patrimoine familial que les fils aînés. Elles avaient donc beaucoup plus de chance que les fils aînés de devenir "maîtresses" du patrimoine familial à cause principalement du départ définitif des fils aînés qui, bien dotés, renonçaient à leurs droits d'aînés.

Tableau 2

L'aînesse intégrale dans la seconde moitié du XIXe siècle : troisième génération

\begin{tabular}{|c|cc|cc|cc|}
\hline Rang de naissance & \multicolumn{2}{|c|}{ Fille } & \multicolumn{2}{c|}{ Fils } & \multicolumn{2}{c|}{ Total } \\
\hline Aîné & 30 & $(63,8 \%)$ & 17 & $(36,2 \%)$ & $\mathbf{4 7}$ & $(\mathbf{5 4 , 7 \%} \%)$ \\
\hline Cadet & $19(48,7 \%)$ & 20 & $(51,3 \%)$ & $\mathbf{3 9}$ & $\mathbf{( 4 5 , 3 \% )}$ \\
\hline Total & $\mathbf{4 9}(\mathbf{5 7 \%} \%)$ & $\mathbf{3 7}(\mathbf{4 3 \%} \%$ & $\mathbf{8 6}(\mathbf{1 0 0 \%})$ \\
\hline
\end{tabular}

Parmi les 39 aînés qui n'ont pas hérité, 15 sont partis en Amérique où parfois un oncle avait émigré avant eux. Avant leur départ, ils ont reçu un dédommagement conséquent pour leur part d'héritage, ce qui leur a permis de financer confortablement leur voyage et leur installation en Amérique. Par ailleurs, 5 aînés, filles ou garçons, ont reçu une dot conséquente pour épouser un héritier ou une héritière aîsé(e). Cette dot constituait un avancement successoral définitif antérieur à leur mariage. Cette solution était souvent appréciée par les aînés qui ne souhaitaient pas se marier tard et qui évitaient ainsi d'attendre le départ définitif de quelques cadets. En outre, grâce à une dot importante, ils épousaient des héritiers ou héritières plus aisés. Après leur 
départ, l'enfant deuxième né devenait l'héritier de la maison. Plus rare, six aînés, filles ou garçons, ont renoncé à leurs droits parce qu'ils refusaient d'assumer les responsabilités d'héritier ou étaient jugés incompétents. Ce n'est pas pour cette raison qu'ils étaient contraints à quitter la maison familiale. Au contraire, ces aînés vivaient célibataires dans la maison jusqu'à la fin de leurs jours. Ainsi, les familles basques semblaient avoir des idées bien définies sur les droits, les responsabilités et les obligations des héritiers, refusant que ces derniers agissent sans le consentement des parents. Effectivement, 8 héritières potentielles n'ont pas hérité car elles avaient conçu leur premier enfant avant le mariage (croyant peut-être forcer les parents à consentir à leur mariage ensuite). Les parents dans ce cas leur retiraient les droits réservés à l'aîné. Enfin, 4 héritiers désignés ont renoncé à l'héritage car ils sont entrés dans les ordres ou ont choisi la carrière de fonctionnaire. Pour toutes les raisons présentées ci-dessus, les filles aînées étaient plus nombreuses à hériter du patrimoine familial que les fils aînés. D'autres raisons justifiaient-elles ces choix ?

\section{Les héritières, aînées de la famille}

Les filles aînées avaient davantage de chance que les fils aînés d'hériter du patrimoine familial pour plusieurs raisons. D'une part, dans la seconde moitié du XIXe siècle, les ressources économiques dans le monde rural basque étaient limitées et ne permettaient à quasiment aucun jeune propriétaire de s'installer dans le village. À partir du Cadastre ${ }^{1}$, l'on se rend compte que le marché de la terre était figé et que les ventes de parcelles de terre étaient rares. Par conséquent, seuls les héritiers et leurs conjoints pouvaient espérer vivre décemment du travail de la terre et de ses revenus dans les villages. Les autres devaient partir.

Par ailleurs, certains aînés renonçaient à l'héritage car ils envisageaient d'autres destins, notamment ceux disponibles sur le marché du travail en milieu urbain. Ils se sont donc installés dans les villes locales ou régionales où les besoins en main d'œuvre artisanale qualifiée étaient grands, notamment à Bayonne dans les métiers liés aux constructions navales. Ce qui a enfin détourné le plus grand nombre d'aînés de l'héritage était avant tout lié aux opportunités offertes en Amérique, l'El Dorado où ils pensaient faire fortune en peu de temps, notamment en Argentine et en Uruguay, mais aussi au Chili, au Mexique, à Cuba, aux Etats-Unis, et au Canada ${ }^{2}$. C'était aussi un moyen pour de nombreux jeunes Basques d'éviter le service militaire. Effectivement, le nombre de déserteurs dans le département des Basses-Pyrénées au XIXe siècle était un des plus élevés en France.

\footnotetext{
'L'analyse systématique du Cadastre dans les six villages étudiés et les villages qui les entourent a démontré que le marché de la terre restait figé et que les nouvelles maisons étaient rares. Dans la seconde moitié du siècle, peu de terres étaient mises en vente et les terres communales n'étaient pas disponibles à la vente. Ainsi, ceux qui n'héritaient pas du patrimoine familial ou qui n'épousaient pas des héritiers ou des héritières ne pouvaient imaginer acquérir des terres et se construire une maison dans le village.

2 Lire l'ouvrage de William A. Douglass \& Jon Bilbao, Amerikanuak. Basques in the New World, Reno, Nevada, University of Nevada Press, 1975. Dans leur recherche, les auteurs ont reconstitué l'historique du parcours des émigrants basques qui d'abord se sont établis en Argentine et en Uruguay (au début et milieu du XIXe siècle), puis au Chili, au Pérou, au Mexique et quelques autres états d'Amérique centrale (dans la seconde moitié du XIXe siècle), et enfin en Amérique du Nord, notamment aux Etats-Unis, à la fin du XIXe siècle et surtout au XIXe siècle.
} 
Il est difficile de déterminer avec certitude si les aînés, filles ou garçons, se retiraient volontairement de la succession, étaient encouragés à quitter la maison ou étaient exclus de l'héritage. Ce qui est certain c'est que quelques filles aînées et beaucoup de fils aînés au XIXe siècle ne respectaient pas les traditions de l'aînesse intégrale. Qui héritait donc à leur place ? Etait-ce l'enfant deuxième né, fille ou garçon, comme nous l'avons constaté dans la première moitié du XIXe siècle? Les filles cadettes avaient-elles alors autant de chance que les fils cadets d'hériter du patrimoine familial ?

Trois conclusions ressortent de l'analyse des reconstitutions de famille et de leurs pratiques successorales dans la seconde moitié du XIXe siècle. En premier lieu, l'héritage unique indéniablement demeurait une pratique récurrente au sein de toutes les familles étudiées en dépit de la mise en place du Code Civil au début du XIXe siècle. Même lorsque les aînés, filles ou garçons, renonçaient à leurs droits d'héritier, les familles s'efforçaient de transmettre la maison et le patrimoine à un seul des enfants. Par conséquent, l'héritage unique a survécu à tous les changements liés à la nouvelle législation apparue après la Révolution Française, notamment celle du partage équitable et égal de tous les biens entre les héritiers imposé par le Code Civil. Pour atteindre cet objectif, les familles ont élaboré de nouvelles stratégies, sacrifiant parfois l'aînesse intégrale, mais faisant en sorte que la maison et les terres soient transmises intégralement à un seul enfant d'une génération à l'autre'

En second lieu, lorsque les propriétaires choisissaient leur héritier parmi les cadets, les filles avaient autant de chance que les garçons d'hériter. Effectivement, pour assurer la succession à la place des 39 aînés, filles ou garçons (mais surtout garçons), qui se sont désistés, les familles ont choisi 19 filles cadettes (soit $48,7 \%$ ) et 20 fils cadets (soit $51,3 \%$ ), pour la plupart des cadets ou cadettes deuxième nés. Ainsi, même si parfois certains propriétaires transmettaient la maison et les terres à leur fille aînée, d'autres n'hésitaient pas à choisir des filles cadettes pour remplacer les aînés qui ont quitté la maison. C'était probablement un honneur d'hériter du titre d'héritier de la maison (etche jaun ou etche jauna) ${ }^{2}$, d'en prendre la responsabilité, et d'en assurer la transmission. La culture familiale traditionnelle était dûment transmise, notamment aux aînés qui connaissaient leurs droits de primogéniture sur les cadets. Lorsque les familles proposaient l'héritage aux cadets, ces derniers, en dépit de leur rang de naissance inférieur, ne résistaient pas. C'était une chance unique qu'ils n'avaient peut-être pas imaginée jusqu'à ce moment.

En troisième lieu, nous avons vu que les familles ne respectaient plus l'aînesse intégrale ou le droit de primogéniture sans distinction de sexe (fille ou garçon). À défaut de l'aîné, ils n'étaient pas non plus forcés de choisir l'enfant deuxième né, fille ou garçon. De plus en plus de propriétaires dans la seconde

\footnotetext{
A titre de comparaison avec d'autres régions de France et d'Europe, il convient de consulter l'ouvrage édité par G. Bouchard, J. Goy \& A.-L. Head-König, Problèmes de la transmission des exploitations agricoles (XVIIIe-XXe siècles), Rome, Ecoles française de Rome, 1998.

2 En basque, le titre d'etche jaun était donné à l'héritier de la maison et celui d'etxeko anderea à l'héritière de la maison. C'était un titre de référence au sein de la communauté villageoise qui non seulement appelait les héritiers et héritières par leur titre mais y ajoutait le norn de la maison, immuable d'une génération à l'autre. Contrairement au nom de famille qui changeait d'une génération à l'autre selon le sexe de l'héritier, le nom de la maison restait inchangé même après sa mise en vente à un étranger de la maison.
} 
moitié du XIXe siècle transmettaient leurs biens à un des enfants, fille ou garçon, aîné ou cadet, ouvrant ainsi la succession à la compétition entre les cohéritiers. Dès lors, elles élaboraient de nouvelles stratégies qui consistaient à accorder les droits d'héritier à un des enfants, premier, second, troisième, quatrième ... né $e^{1}$. Il était peut-être souhaitable de transmettre le patrimoine familial à un cadet, fille ou garçon, afin d'éviter les mariages tardifs, le long célibat de l'aîné forcé d'attendre le départ de certains de ses cadets pour se marier et s'établir dans la maison avec le conjoint. Lorsque l'aîné renonçait à ses droits, il donnait la possibilité à ses parents de choisir l'héritier parmi les ènfants les plus aptes, les plus dociles, voire même les plus soumis pour assurer les responsabilités de la transmission. Il est évident que les filles tenaient une place de choix dans ces critères. Ils n'avaient parfois pas le choix car les fils émigraient en Amérique. Les filles étaient les dernières à résider dans la maison et les plus disponibles à perpétuer les traditions familiales. Quoi qu'il en soit, les héritières (soit $57 \%$ des cas) étaient probablement préférées aux héritiers (soit $43 \%$ des cas) pour leur docilité, leur obéissance et leur attachement aux traditions (voir Tableau 2). En outre, par ce choix, les parents étaient quasiment certains que leur autorité parentale n'allait probablement jamais être remise en cause par une héritière, ni même par un gendre, tous deux soumis aux parents donateurs qui dès lors gardaient le contrôle de l'exploitation jusqu'à leur décès.

La question que nous devons nous poser est la suivante : était-ce si honorable au XIXe siècle de devenir héritier de la maison lorsque les jeunes héritiers ou héritières ne pouvaient disposer du patrimoine comme ils le souhaitaient et qu'ils devaient travailler toute leur vie pour récolter les sommes suffisantes pour dédommager les cohéritiers de leur part de l'héritage, comme la loi l'oblige? Il semble que le statut d'héritier ou d'héritière demeurait enviable au XIXe siècle. Mais ce statut présentait quelques inconvénients. L'héritier ne pouvait se marier comme il le souhaitait. Il devait épouser une personne capable d'apporter une dot suffisamment importante pour dédommager un ou plusieurs cohéritiers et qui convienne aux parents. En outre, l'héritier devait parfois attendre plusieurs années avant de se marier, le temps que plusieurs co-héritiers quittent la maison et laissent leur place à la jeune famille. Enfin, les parents vivaient parfois longtemps et le jeune héritier et son conjoint devaient se soumettre à leur autorité et obéir à leurs décisions jusqu'à ce qu'ils prennent leur retraite ou décèdent. Le rôle d'héritier par conséquent ne présentait pas que des avantages, ce qui peut expliquer pourquoi certains ont choisi d'autres destins, notamment les aînées issues de familles de petits propriétaires qui ne possédaient que quelques ares de terres et dont les droits d'héritier ne leur apportaient que labeur et privation. C'est pourquoi les pratiques de l'héritage unique variaient considérablement selon les villages, la taille de la propriété à hériter, et le marché du travail.

\section{Pratiques différenciées selon les villages et les propriétaires}

Nous pouvons certes émettre des conclusions sur les comportements des familles et le choix des héritiers. Cependant, ces généralités ne s'appliquent pas exactement de la même manière selon les villages et les propriétaires.

1 Sur les destins des cadets en France, il convient de se référer à l'ouvrage édité par Gérard Bouchard, John A. Dickinson \& Joseph Goy, Les exclus de la terre en France et au Quebec (XVlle-XXe siècles), Sillery (Quebec), Septentrion, 1998. 
Effectivement, dans certains villages, tels que Mendive et Isturits en Basse Navarre, les pratiques successorales des familles demeuraient proches de celles de l'Ancien Régime, la plupart respectant les traditions de l'aînesse intégrale en transmettant tout leur patrimoine à l'aîné des enfants, fille ou garçon, notamment dans la première moitié du XIXe siècle. Mendive était un village de montagne prospère, aux revenus agricoles élevés, proche de Saint-Jean-Pied-dePort, ou les exploitants pouvaient écouler leur surplus agricole et leurs bestiaux. Les exploitations étaient rentables et lorsque les aînés le pouvaient, ils acceptaient la succession. C'est pourquoi les aînés, filles ou garçons, renonçaient rarement à leurs droits d'héritiers, même dans la seconde moitié du XIXe siècle. La plupart des héritiers et des héritières étaient les fils et les filles aînées de la famille. De même, à Isturits, un village de plaine où les propriétaires agricoles étaient peu nombreux mais aisés. Les héritiers héritaient donc d'une exploitation très rentable et d'un statut social honorable au sein de la communauté. C'est pourquoi, là aussi, les aînés renonçaient rarement à leurs droits et à leur statut d'héritier pendant tout le siècle. Lorsque, à l'occasion, les héritiers n'acceptaient pas la succession, les familles choisissaient un fils cadet plutôt qu'une fille cadette, s'assurant que ce fils héritier soit travailleur et prenne grand soin de l'exploitation familiale et de sa transmission à la génération suivante. Dans ces deux villages prospères où les propriétaires agricoles étaient aisés, par conséquent, les pratiques de l'aînesse intégrale restaient intactes pendant toute la période.

Les comportements des propriétaires étaient totalement différents dans les villages pauvres et isolés dans les montagnes où les exploitations agricoles étaient pour la plupart de petite taille et parfois de taille moyenne, mais où tous les enfants ne pouvaient espérer s'établir. Les hommes n'hésitaient pas à quitter la maison familiale pour aller s'installer en ville ou en Amérique. C'est pourquoi les familles choisissaient leur héritier parmi les filles, première née ou cadette. C'est le cas des Aldudes en Basse Navarre et d'Alçay en Soule. Effectivement, les parents encourageaient les fils au départ définitif de la maison, profitant d'un petit dédommagement successoral pour s'installer ailleurs, notamment en Amérique. C'est ainsi qu'à mesure que le temps passait, de plus en plus de fils aînés, accompagnés de leurs frères cadets, émigraient en Amérique, en renonçant à leurs droits d'aînés et faisant bénéficier leurs sœurs cadettes. Aux Aldudes, aucun des fils aînés n'a pris la succession de l'exploitation familiale dans la première moitié du XIXe siècle car ils ont tous émigré en Amérique (en Argentine ou en Uruguay généralement, mais aussi au Mexique et en Amérique $\mathrm{du}$ Nord à la fin du siècle). L'Amérique offrant des opportunités professionnelles à mobilité sociale asccndante, de plus en plus de fils aînés ou cadets originaires des Aludes émigraient, laissant l'exploitation familiale à une des sceurs, aînées ou cadettes. Cette situation a persisté pendant tout le siècle car les opportunités en Amérique étaient de plus en plus grandes, notamment dans l'élevage et l'artisanat, et cela leur permettait dans le même temps d'éviter le service militaire. Les hommes d'Alçay n'ont commencé à émigrer en Amérique que dans la seconde moitié du siècle. C'est pourquoi dans la première moitié du siècle, peu de fils aînés ont renoncé à leurs droits. Cependant dans la seconde moitié du siècle, la plupart des filis aînés a renoncé à la succession, préférant émigrer en Amérique, laissant alors la responsabilité de l'exploitation à une des scurs cadettes. C'est dans les villages (souvent pauvres et isolés) où l'émigration était la plus forte et la plus précoce dès le début du XIXe siècle que nous avons constaté la présence d'un grand nombre d'aînés ayant renoncé à leurs droits d'héritier. Ces derniers se sont très tôt détournés des pratiques ancestrales 
de l'aînesse intégrale. Dans ces circonstances seulement, les filles, aînées ou cadettes, héritaient de la maison et des terres à la place des fils.

Entre les villages qui respectaient scrupuleusement les pratiques de l'aînesse intégrale (Mendive et Isturits) et ceux qui s'en détournaient totalement (aux Aldudes et Alçay), il y avait d'autres villages, ceux qui indifféremment choisissaient leurs héritiers parmi les aînés ou les cadets, filles ou garçons. C'était le cas de Sare en Labourd et Amendeuix en Basse Navarre. Sare était un village aisé sur le flan ouest des Pyrénées, près de plusieurs marchés locaux, dont celui de Saint-Jean-de-Luz. Les propriétaires, relativement aisés, respectaient rigoureusement les pratiques de l'aînesse intégrale, transmettant les biens de famille à l'aîné des enfants, fille ou garçon, dans la première moitié du siècle. Cependant, l'Amérique commença à attirer les jeunes surtout après 1860 , ce qui, dans la seconde moitié du siècle, contraignit les propriétaires à choisir leurs héritiers parmi tous leurs enfants, aînés ou cadets, filles ou garçons. $\dot{A}$ Amendeuix, enfin, les quelques propriétaires n'ont transmis leurs biens qu'à des filles cadettes dès la première moitié du siècle. À la génération suivante, les propriétaires ont transmis leurs biens à l'aîné des enfants, généralement des filles ou bien alors aux fils cadets. Quoiqu'il en soit, à Sare comme à Amendeuix, les familles ne respectaient qu'en partie les traditions de l'aînesse intégrale, $50 \%$ étant des aînés, filles ou garçons, et $50 \%$ des cadets, filles ou garçons.

Des observations ci-dessus, nous pouvons déduire qu'au cours du XIXe siècle, de plus en plus de fils aînés renonçaient à leurs droits d'héritier et par conséquent laissaient leur place à des frères ou à des sœurs cadettes. Les raisons de cette évolution dans les comportements des aînés sont multiples : les opportunités économiques limitées dans les campagnes du Pays Basque au XIXe siècle, l'industrialisation et l'urbanisation en France, le développement de la fonction publique, mais aussi les opportunités en Amérique, notamment en Argentine et en Uruguay (et plus tard au Mexique et en Amérique du Nord), une façon de faire fortune peut-être et d'éviter le service militaire. C'est dans ces circonstances que de plus en plus de femmes, aînées ou cadettes, ont hérité du patrimoine familial, ce qui convenait parfois aux parents qui pouvaient se permettre de choisir la fille la plus volontaire, docile et soucieuse des traditions pour leur succéder. Il est impossible de dire si les parents choisissaient une fille par nécessité (le départ définitif de tous les fils aînés ou cadets) ou par choix (une fille cadette et un gendre se montrant plus obéissants). Ce qui est certain c'est que, dans leurs pratiques de l'héritage unique, ils étaient prêts à sacrifier les pratiques ancestrales de l'aînesse intégrale, soucieux de transmettre les biens à un seul enfant et de protéger la viabilité économique de l'exploitation familiale d'une génération à l'autre. Cette situation n'était possible qu'avec l'accord de tous les cohéritiers que l'héritier dédommageait d'une somme d'argent, une compensation inférieure à leur part légale de l'héritage mais suffisante pour s'établir comme gendre ou belle-fille dans une autre propriété de la commune, ou partir s'installer en ville ou en Amérique.

Les conséquences de cette évolution est le départ de beaucoup d'hommes et de femmes originaires des villages ruraux du Pays Basque. Le départ de tous ces cohéritiers a nécessité la mise en place de nouvelles stratégies relatives aux modalités de transmission du patrimoine, qui obligeaient les familles à dédommager plus ou moins équitablement tous les cohéritiers afin que certains épousent des héritiers et d'autres s'installent ailleurs dans le milieu rural, en ville 
ou en Amérique. L'analyse de ces modalités successorales fera l'objet d'une étude ultérieure. Quoi qu'il en soit, les cohéritiers y trouvaient probablement leur compte, voulant éviter la mise en vente du patrimoine familial, qui, après remboursement des frais ne leur apporterait pas forcément davantage que la dot qu'ils avaient perçue. Le départ des cohéritiers affectait inévitablement les structures familiales. Contrairement à ce qu'a affirmé Frédéric Le Play, la famille souche n'était pas en voie de disparition. Bien au contraire, les parents cohabitaient avec l'héritier, son conjoint et leurs enfants jusqu'à leur décès. Cependant, les structures familiales étaient rarement aussi complexes que dans le passé car rares étaient les cohéritiers célibataires qui au XIXe siècle cohabitaient dans la maison familiale après le mariage de l'héritier ou de 'l'héritière. Dans ce cas, les structures complexes de la famille souche n'existaient que lors de cette cohabitation, une cohabitation plus ou moins longue selon la longévité des parents ${ }^{1}$. Au décès des parents anciens, la famille ne se composait plus que du couple, dont l'héritier et son conjoint, et leurs enfants et ce, jusqu'à ce que l'héritier de la génération suivante soit autorisé à se marier et à s'établir dans la maison ${ }^{2}$.

\section{Conclusion}

L'aînesse intégrale, cette pratique successorale traditionnelle qui avait pour but de préserver la maison et les terres intactes et de les transmettre intégralement à l'aîné des enfants, fille ou garçon, est devenue une pratique de moins en moins courante à la fin du XIXe siècle. Bien que souhaitées par les familles, les coutumes ne pouvaient plus être mises en pratique de la même manière que pendant l'Ancien Régime. En fait, les biens étaient transmis indifféremment à un fils ou une fille, qu'il soit aîné ou cadet. Vers la fin du siècle, les filles, aînées ou cadettes, avaient parfois même plus de chance que les fils d'hériter de la maison et des terres. Grâce à elles, l'exploitation familiale restait viable et était transmise intégralement à la génération suivante. Les fils étaient parfois si nombreux à quitter la maison ou le village qu'il était peut-être difficile de trouver un successeur. Au lieu de cela, ils préféraient épouser une héritière plus aisée ou émigrer en Amérique. Heureusement, les filles cadettes étaient disponibles pour assurer les responsabilités et la succession de l'exploitation en l'absence des fils émigrés en Amérique. Quoi qu'il en soit, les familles ont préservé l'aspect vital des pratiques ancestrales, l'héritage unique, en préservant le patrimoine intact d'une génération à l'autre. Bien que l'aînesse intégrale ne soit plus le modèle unique de transmission du patrimoine au XIXe siècle, l'héritage unique par les filles, aînées ou cadettes, était devenu une pratique particulièrement répandue, notamment dans certains villages tels que Les Aldudes et Alçay où les exploitations étaient généralement petites, les possibilités d'expansion économique limitées, et l'émigration en Amérique très répandue. Les garçons émigraient pour faire fortune et améliorer leurs conditions de vie ou pour éviter le service militaire obligatoire, voire les deux raisons à la fois. S'ils n'émigraient pas, ils épousaient généralement une héritière plus aiséc qu'eux, issue d'une maison plus grande que celle de leurs parents, et

1 Voir les arguments que Lutz Berkner a développés dans son article à propos de la famille souche dans le cycle de vie des familles autrichiennes: "The stem-family and the developement of the peasant household: an eighteenth-century Austrian example", American Historical Review, 1972, 77, 2, p. 398-418.

2 A ce propos, voir article de Marie-Pierre Arrizabalaga, "The stem family in the French Basque country: Sare in the nineteenth century", Journal of Family History, Vol. 22, 1, 1997, p. 50-69. 
amélioraient ainsi leur statut dans le village. Quoi qu'il en soit, de moins en moins d'hommes héritaient du patrimoine familial, préférant laisser à une de leurs scurs le soin d'assurer les responsabilités de la maison, des terres et de l'héritage. Les fils allaient chercher ainsi le moyen d'améliorer leurs conditions de vie dans le village (comme gendre), en ville (comme artisans) ou à l'étranger. C'était une aubaine pour les filles, notamment les cadettes, qui sans cela n'auraient jamais eu l'occasion d'hériter de la maison et des terres. Les parents eux gardaient le contrôle de la maison pendant toute leur vie, certains que les filles et leurs conjoints obéiraient à leurs ordres jusqu'à la transmission définitive des biens familiaux (à leur décès).

Ainsi, les familles ont su utiliser les coutumes et le Code Civil pour mettre en place de nouvelles stratégies afin de préserver l'héritage unique, sacrifiant néanmoins les pratiques ancestrales de l'aînesse intégrale. Ce qui est certain, c'est que les parents gardaient le contrôle de la maison et des terres jusqu'à leur décès en attendant de trouver des solutions pour éviter le partage égalitaire des biens entre les cohéritiers. C'était le rôle des jeunes héritiers de trouver le moyen de dédommager les frères et les soeurs qui ne percevaient pas un dédommagement équitable pour leur part d'héritage, mais suffisant pour qu'ils puissent s'installer ailleurs. La maison devait être préservée coûte que coûte, même si parfois cela passait par l'absence de dédommagement. Lorsque les hommes et les femmes avaient l'occasion d'épouser une héritière ou d'hériter, ils acceptaient, les hommes pour améliorer leurs conditions en épousant une héritière plus aisée et les femmes en héritant d'une maison et de terres et donc d'un statut qu'elles n'auraient jamais pu avoir si les fils aînés ne s'étaient pas désistés à leur profit. Ces pratiques de l'héritage unique, sans distinction de sexe et de rang de naissance, mettaient certes en péril les coutumes ancestrales de l'aînesse intégrale, mais elles semblaient satisfaire tout le monde, les parents, les fils et les filles à la fois, qui y trouvaient leur compte tout en assurant l'indivisibilité de la maison et des terres appartenant à la famille depuis plusieurs générations.

\section{Bibliographie}

Abelson, Andrew, "Inheritance and population control in a Basque valley before 1900", Peasant Studies, 1978, 7, 1, 11-27.

Arrizabalaga, Marie-Pierre, "Comment le marché de l'emploi national et international a-t-il influencé les destins individuels au sein des familles basques et les modalités de transmission du patrimoine au XIX siècle ?", à paraître en 2002, 19 pages.

"Basque women and migration in the nineteenth century", The History of the Family. An International Quarterly, à paraître en 2002, 22 pages.

"Les Basques dans l'Ouest américain, 1900-1910", Lapurdum, 2000, $\mathrm{V}, 335-350$.

,Famille, succession, émigration au Pays Basque au XIXe siècle. Etude des pratiques successorales et des comportements migratoires au sein de familles basques, Thèse de troisième cycle, Ecole des Hautes Etudes en Sciences Sociales, 1998, 433 pages.

"The stem family in the French Basque Country : Sare in the nineteenth century", Journal of Family History, 1997, 1, 50-69.

, "Structures familiales et destins migratoires à Sare au XIXe siècle", Lapurdum, 1997, II, 237-255. 
"Réseaux et choix migratoires au Pays Basque. L'exemple de Sare au XIXe siècle", Annales de démographie historique, 1996, 423-446.

, Family structures, inheritance pratices, and migration networks in the Basses-Pyrénées in the nineteenth century : Sare, Thèse de doctorat en Histoire, Université de Californie, Davis (USA), 1994, 351 pages.

Assier-Landrieu, Louis (dir.), Une France coutumière. Enquête sur les "usages locaux" et leur codification (XIXe-XXe siècles), Paris, Éditions du CNRS, 1990.

, "Coutume savante et droit rustique. Sur la légalité paysanne", Etudes rurales, 110-112, 1988, 119-175.

Le Peuple et la loi. Anthropologie historique des droits paysans en Catalogne française, Paris, Librairie de droit et de jurisprudence, 1987.

Coutume et rapports sociaux. Etude anthropologique des communautés paysannes du Capcir, Paris, Editions du CNRS, 1981.

Barcelo, Ramón, "Transmission héréditaire et systèmes de production : le cas de la Soule (Pyrénées-Atlantiques)", Sociologie du travail, 1988, 3, 443-460.

Baronnies des Pyrénées. (Les) Maisons, mode de vie, société, Tome I, Dirigé par I. Chiva \& J. Goy, Paris, EHESS, 1981.

Baronnies des Pyrénées. (Les) Maisons, espace, famille, Tome II, dirigé par I. Chiva \& J. Goy, Paris, EHESS, 1986.

Berkner, Lutz K, "The stem-family and the developmental cycle of the peasant household : an eighteenth-century Austrian example", American Historial Review, 1972, 77, 2, 398-418.

Bobinska, Celina \& Goy, Joseph (dir,), Les Pyrénées et les Carpates, XVIe-XXe siècles, Warszawa, Editions scientifiques de Pologne, 1981.

Bonnain, Rolande, "Houses, heirs and non-heirs in the Adour Valley. Social and geographic mobility in the nineteenth century", The History of the Family : an International Quarterly, 1 (3), 1996, 273-296.

Bonnain, Rolande \& Bouchard, Gérard \& Goy, Joseph (sous la direction de), Transmettre, hériter, succéder. La reproduction familiale en milieu rural : France - Québec, XVIIIe - XXe siècles, Paris, EHESS, 1992.

Bouchard, Gérard, Goy, Joseph \& Head-Konig, Anne-Lise (eds), Problèmes de la transmission des exploitations agricoles (XVIIIe - XXe siècles), Rome, Editions de l'Ecole Française de Rome, 1998.

Bouchard, Gérard, Dickinson, John A. \& Goy, Joseph, Les Exclus de la terre en France et au Quebec (XVIIe - XXe siècles), Paris, Septentrion, 1998.

Bourdieu, Pierre, "Célibat et condition paysanne", Etudes rurales, 5-6, 1962, $32-135$.

"Les stratégies matrimoniales dans les systèmes de reproduction", Annales ESC, 4-5, 1972, 1105-1127.

Burguière, André, "Pour une typologie des formes d'organisation domestique de I'Europe moderne (XVVIe - XIXe siècles)", Annales E.S.C., 3, 1986, 639655.

Collomp, Alain, "From stem family to nuclear family : changes in the coresident domestic group in Haute-Provence between the end of the eighteenth and the middle of the nineteenth centuries", Continuity and Change, 1988, 3, 1, 65-81.

Coûtumes générales, gardées et observées au Païs et baillage de Labourt, et reffort d'icelui, Bordeaux, J-B Lacornée, 1760.

Derouet, Bernard, "Transmettre la terre. Origines et inflexions récentes d'une problématique de différence", Histoire et sociétés rurales, 1994, 2, 33-67. 
"Le partage des frères. Héritage masculin et reproduction sociale en Franche-Comté aux XVIIIe et XIXe siècles", Annales ESC, 2, 1993, 453474.

" "Pratiques successorales et rapport à la terre : les sociétés paysannes d'ancien régime", Annales ESC, 1, 1989, 173-206.

Douglass, William A., "The Basque stem-family household : myth or reality?", Journal of Family History, 1988, 73, 1, 75-89.

Douglass, William A. \& Bilbao, Jon, Amerikanuak. Basques in the New World, Reno, University of Nevada Press, 1975.

Duroux, Rose, Les Auvergnats de Castille. Renaissance et mort d'une migration au XIXe siècle, Clermont-Ferrand, Association des Publications de la Faculté des Lettres et Sciences Humaines, 1992, 1995.

Etcheverry, Louis, "Les coutumes successorales du Pays Basque au XIXe siècle", in La Tradition au Pays Basque, Paris, Bureaux de la tradition nationale, 1899 , p. 179-190.

, "L'émigration dans les Basses-Pyrénées pendant soixante ans", Revue des Pyrénées, 1893, 509-520.

'L'émigration des Basses-Pyrénées en Amérique", Réforme sociale, $1886,490-514$.

Fauve-Chamoux, Antoinette, "Les frontières de l'autorégulation paysanne : croissance et famille-souche", Revue de la bibliothèque nationale, 1993, $50,38-47$

,"Household forms and living standards in preindustrial France : from models to realities", Journal of Family History, 1993, 18, 2, 135-156.

"Le fonctionnement de la famille-souche dans les Baronnies des Pyrénées avant 1914", Annales de démographie historique, 1987, 241-262.

"Les structures familiales au Royaume des familles-souches Esparros", Annales ESC, 1984, 3, 514-528.

Fine-Souriac, Agnès, "La famille-souche pyrénéenne au XIXe siècle. Quelques réflexions de méthode", Annales ESC, 1977, 3, 478-487.

Fougères, Alain, Les Droits de famille et les successions au Pays Basque et en Béarn d'après les anciens textes, Bergerac, H. Trillaud, 1938.

Goyheneche, Jean, For et coutumes de Basse-Navarre, Bayonne, Elkar, 1985.

Grosclaude, Michel, La Coutume de la Soule. Traduction, notes et commentaires, Saint-Etienne-de-Baïgorry, Izpegi, 1993.

Lacanette-Pommel, Christine, Les Béarnais et le Code Civil. Etude des pratiques successorales et matrimoniales dans les Pyrénées (1789-1840), Thèse de troisième cycle en Droit, Université des Sciences Sociales de Toulouse, 1998.

Lafourcade, Maïte, Mariages en Labourd sous l'Ancien Régime. Les contrats de mariage du pays de Labourd sous le règne de Louis XVI (Etude juridique et sociologique), Bilbao, Universitad del País Vasco, 1989.

Lamaison, Pierre, "La diversité des modes de transmission : une géographie tenace", Etudes rurales, 1988, 110-111-112, 119-175.

Laslett, Peter \& Wall, Richard (eds), Household and Family in Past Time. Comparative studies in the size and structure of the domestic group over the last three centuries, Cambridge, Cambridge University Press, 1972.

Laslett, Peter \& Wall, Richard \& Robin, Jean (eds.), Family Forms in Historical Europe, Cambridge, Cambridge University Press, 1983.

Le Play, Frédéric, La Réforme sociale en France déduite de l'observation comparée des peuples européens, 3 volumes, Paris, Dentu, 1878.

L'Organisation de la famille selon le vrai modèle signalé par l'histoire de toutes les races et de tous les temps (Paris: Tequi, 1871). 
Le Roy Ladurie, Emmanuel, "Système de la coutume. Structures familiales et coutume d'héritage en France au XVIe siècle", Annales E.S.C., 4-5, 1972, 825-846.

Moch, Leslie Page, Paths to the City : Regional Migration in NineteenthCentury France, Beverly Hills, Sage Publications, 1983.

O'Quin, P., Dii Décroissement de la population dans le département des BassesPyrénées, Pau, Imprimerie Vignancour, 1856.

Peyronnet, Jean-Claude, "Famille élargie ou famille nucléaire ? L'exemple du Limousin au début du XIXe siècle", Revue d'histoire moderne contemporaine, $1975,568-582$.

Poumarède, Jacques, "Famille et ténure dans les Pyrénées du Moyen Age au XIXe siècle", Annales de démographie historique, 1979, 347-360. , Les Successions dans le Sud-Ouest de la France au Moyen Age, Paris, Presses Universitaires de France, 1972.

Rosental, Paul-André, Les Sentiers invisibles. Espace, famille et migrations dans la France du $19^{2}$ siècle, Paris, EHESS, 1999.

Saint-Macary, Jacques, La Désertion de la terre en Béarn et dans le Pays Basque, Pau, Lescher-Moutoué, 1939.

Segalen, Martine, Quinze générations de Bas-Bretons. Parenté et Société dans le Pays Bigouden Sud, 1720-1980, Paris, Presses Universitaires de France, 1985.

Thirsk, Joan \& Thompson, E.P. \& Goody, Jack, Family and Inheritance : Rural Society in Western Europe, 1200-1800, Cambridge, Cambridge University Press, 1976.

Verdon, Michel, "Rethinking complex households : the case of the Western Pyrenean 'Houses"', Continuity and Change, 1996, 11, 2, 191-215.

"The stem family : toward a general theory", Journal of Interdisciplinary History, 1979, X, 1, 87-105.

Yver, Jean, Egalité entre héritiers, et exclusion des enfants dotés : essai de géographie coutumière, Paris, Éditions Sirey, 1966.

Zink, Anne, L'Héritier de la maison. Géographie coutumière du Sud-Ouest de la France sous l'Ancien Régime, Paris, EHESS, 1993. 